\title{
Comparison between linear elasticity and large elastic deformation in the study of the contraction of the myocardium
}

\author{
R. M. Shoucri \\ Department of Mathematics and Computer Science, \\ Royal Military College Canada, Kingston, Ontario, Canada
}

\begin{abstract}
The purpose of this study is to compare the mathematical formalism obtained by using linear elasticity with the formalism obtained by using large elastic deformation in the calculation of the stress in the myocardium. This study is motivated by previous results based on the theory of linear elasticity that have shown consistency in the calculation of the stress expressed as a sum of an active and a passive component. The myocardium is represented as a thick-walled elastic cylinder contracting symmetrically in which the active force is expressed as a force/unit volume of the myocardium.
\end{abstract}

Keywords: cardiac mechanics, mathematical modelling of ventricular contraction, pressure-volume relation, active force of the myocardium.

\section{Introduction}

One of the difficulties encountered in the study of stress-strain analysis in the myocardium is the fact that the myocardium is a composite structure with active elements generating an active force during the contraction of the myocardium [1-6]. An approach to solve this problem was to model the active force generated by the myocardium as force per unit volume of the myocardium. By using large elastic deformation, this approach was applied to the study of the pressure-volume relation in the left ventricle $[1,2]$. By using the linear theory of elasticity, the concept of active force/unit volume of the myocardium was used to derive expressions for the active stress due to the muscular fibres and the passive stress resulting from the deformation of the passive medium of the myocardium 
[3-6]. A review of various clinical applications obtained by introducing the concept of active force/unit volume of the myocardium in the formalism describing the pressure-volume relation in the right and left ventricles is given in [7].

The consistency of the results obtained in the calculation of active and passive stress in the myocardium by using the concept of active force/unit volume of the myocardium suggests a possible investigation of the relation between the mathematical formalism based on linear elasticity [3-6] and the mathematical formalism based on large elastic deformation for the calculation of the stress $[1,2]$. It is shown in what follows that it is possible to link the two approaches together. A review of other methods for the calculation of the stress in the myocardium based on the theory of linear elasticity can be found in [8].

\section{Mathematical formalism}

\subsection{Stress in the myocardium}

The myocardium is represented as a thick-walled cylinder contracting symmetrically. According to [9] and as explained in the appendix of [1], the total stress $t_{i j}$ induced in the myocardium can be expressed as the result of the stress $\sigma_{i j}$ induced by the deformation of the passive medium of the myocardium and the fibre generated stress $q_{i j}$, with $t_{i j}=\sigma_{i j}-q_{i j}$. In a symmetric contraction the stress components depend only on the radial variable $r$. By assuming a quasi-static equilibrium (inertia and viscous forces neglected), the components $t_{i j}$ of the stress must satisfy the local equilibrium equation in the radial direction, which is expressed as follows in cylindrical coordinates (see [1])

$$
\frac{\partial t_{r}}{\partial r}+\frac{t_{r}-t_{\theta}}{r}=0
$$

We have written $t_{r}=t_{r r}$ and similarly for other components. By substituting $t_{i j}=$ $\sigma_{i j}-q_{i j}$ we get

$$
\frac{\partial \sigma_{r}}{\partial r}+\frac{\sigma_{r}-\sigma_{\theta}}{r}-D_{r}=0
$$

As in [1] we have $q_{r}=0$ and $D_{r}=-q_{\theta} r$, where the radial active force $D_{r}$ per unit volume of the myocardium appearing in eqn (2) is shown in fig. 1. Eqns (1) and (2) are valid in both linear and non-linear elasticity. The stress $\sigma_{i j}$ is related to the deformation of the passive medium of the myocardium by the constitutive equations as shown in what follows. 


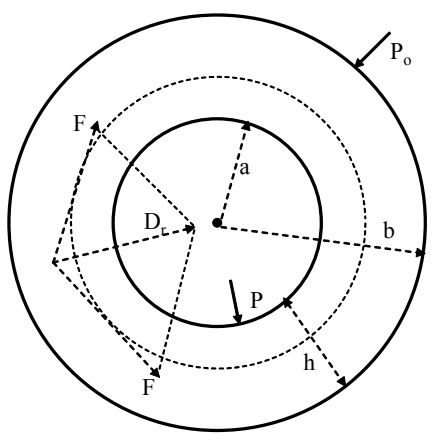

Figure 1: Cross-section of a thick-walled cylinder representing the left ventricle. A helical fibre is projected on the cross-section along the dotted circle shown in figure. Because of the assumed axisymmetric contraction, an active radial force $D_{r}$ is generated during the contraction phase. $P=$ left ventricular pressure, $P_{o}=$ outer pressure on the pericardium, $a=$ inner radius, $b=$ outer radius, $h=b-a=$ thickness of the myocardium.
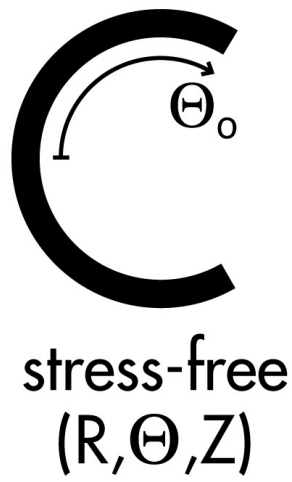
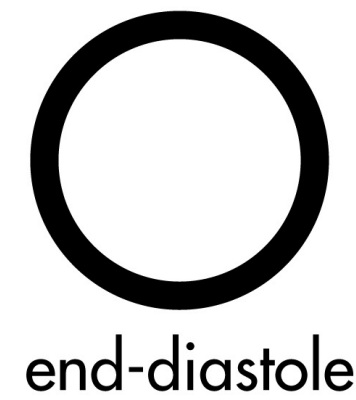

$\left(r_{\text {ed }}, \theta_{\text {ed }}, z_{\text {ed }}\right)$

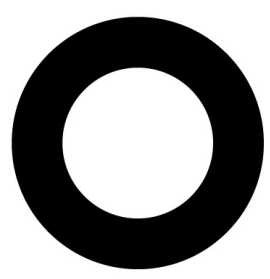

contraction $(r, \theta, z)$

Figure 2: Cross-section of a thick-walled cylinder. The left-hand side represents the stress-free configuration, the middle configuration corresponds to end-diastole and the right-hand side the configuration during the contraction phase.

\subsection{Stretch ratios}

As shown in fig. 2 the myocardium will change from a stress free configuration described in cylindrical coordinates by $(R, \Theta, Z)$, to a configuration at enddiastole described by $\left(r_{e d}, \theta_{e d}, z_{e d}\right)$ and finally to a configuration described by $(r$, $\theta, z)$ in the contraction phase. We define the stretch ratios as 


$$
\Lambda_{1}=\frac{d r}{d R} \quad \Lambda_{2}=\frac{r d \theta}{R d \Theta} \quad \Lambda_{3}=K=\frac{d z}{d Z}
$$

It is assumed that $\theta_{e d}=\left(\pi / \Theta_{0}\right) \Theta$ where $\Theta_{o}$ is the opening angle in the stress-free configuration shown in fig. 2 and $r=r(R)$. It is assumed that the passive medium surrounding the muscular fibres is incompressible, one can then write the incompressibility condition

$$
\Lambda_{1} \Lambda_{2} \Lambda_{3}=1
$$

We shall need in what follows to express our results referred to the configuration at end-diastole; this is why we introduce the stretch ratios

$$
\lambda_{1}=\frac{d r}{d r_{e d}} \quad \lambda_{2}=\frac{r d \theta}{r d \theta_{e d}} \quad \lambda_{3}=k=\frac{d z}{d z_{e d}}
$$

Because of the assumed axisymmetric contraction of the myocardium we can write $\theta=\theta_{e d}, r_{e d}=r_{e d}(R)$ and $r=r\left(r_{e d}\right)$. We shall also need the radial contraction ratio $\mu=r / r_{e d}$ and the incompressibility condition

$$
\lambda_{1} \lambda_{2} \lambda_{3}=1
$$

By using eqns (5) and by assuming that $k$ is constant, eqn (6) can be integrated in the form

$$
k r^{2}=r_{e d}^{2}-C_{k}, \quad k a^{2}=a_{e d}^{2}-C_{k}, \quad k b^{2}=b_{e d}^{2}-C_{k}
$$

with $a=$ inner radius, $b=$ outer radius of the cylinder and $C_{k}$ is a constant. The Lagrangian strain components $E_{i j}$ are expressed in cylindrical coordinates as follows

$$
\begin{aligned}
& E_{R R}=1 / 2\left(\Lambda_{1}^{2}-1\right) \quad E_{\Theta \Theta}=1 / 2\left(\Lambda_{2}^{2}-1\right) \\
& E_{Z Z}=1 / 2\left(\Lambda_{3}^{2}-1\right)
\end{aligned}
$$

We also note the following relations that we shall need in what follows

$$
\Lambda_{2}=\frac{r d \theta}{R d \Theta}=\frac{r d \theta}{r_{e d} d \theta_{e d}} \frac{r_{e d} d \theta_{e d}}{R d \Theta}=\mu \frac{r_{e d} d \theta_{e d}}{R d \Theta}
$$




$$
K=\frac{d z}{d Z}=k \frac{d z_{e d}}{d Z}
$$

We shall also need for the constitutive relations the strain invariant

$$
I=\Lambda_{1}^{2}+\Lambda_{2}^{2}+\Lambda_{3}^{2}
$$

So far the relations derived apply to both large elastic deformations and linear elasticity. What makes the difference between these two approaches is the way the stress and the strain are related together. This is what is called the constitutive equations discussed in what follows.

\section{Large elastic deformation}

\subsection{Constitutive relations}

It is assumed that the tissues of the myocardium will exhibit a property of transverse isotropy with respect to the Z-axis as discussed in [10]. In this case the strain energy function $W$ can be expressed as

$$
W \equiv W(I, K)
$$

Because of the assumed axisymmetric contraction $K$ is constant and the stress generated as a result of the deformation of the passive medium of the myocardium can be expressed as in [1]

$$
\begin{gathered}
\sigma_{r}=\Lambda_{1} \frac{\partial W}{\partial \Lambda_{1}}-p_{h}=\frac{2}{\Lambda_{2}^{2} K^{2}} \frac{\partial W}{\partial I}-p_{h} \\
\sigma_{\theta}=\Lambda_{2} \frac{\partial W}{\partial \Lambda_{2}}-p_{h}=2 \Lambda_{2}^{2} \frac{\partial W}{\partial I}-p_{h} \\
\sigma_{z}=\Lambda_{3} \frac{\partial W}{\partial \Lambda_{3}}-p_{h}=2 K^{2} \frac{\partial W}{\partial I}-p_{h}
\end{gathered}
$$

where $p_{h}$ is a pressure resulting from the incompressibility condition. By making use of eqns (9) and (10), eqns (13)-(15) can be written in the form

$$
\sigma_{r}=\frac{2}{\mu^{2} k^{2}} \frac{\partial W}{\partial I}\left(\frac{d r_{e d}}{d R}\right)^{2}-p_{h}
$$


8 Modelling in Medicine and Biology VII

$$
\begin{gathered}
\sigma_{\theta}=2 \mu^{2} \frac{\partial W}{\partial I}\left(\frac{r_{e d}}{R} \frac{d \theta_{e d}}{d \Theta}\right)^{2}-p_{h} \\
\sigma_{z}=2 k^{2} \frac{\partial W}{\partial I}\left(\frac{d z_{e d}}{d Z}\right)^{2}-p_{h}
\end{gathered}
$$

\subsection{Calculation of the radial stress}

By subtracting eqns (16) and (17) we get by writing $W_{I}=\partial W / \partial I$

$$
\begin{aligned}
& \sigma_{\theta}-\sigma_{r}=2 W_{I} \times \\
& \left\{\mu^{2}\left[\left(\frac{r_{e d}}{R} \frac{d \theta_{e d}}{d \Theta}\right)^{2}-\left(\frac{d r_{e d}}{d R}\right)^{2}\right]+\left(\mu^{2}-\frac{1}{\mu^{2} k^{2}}\right)\left(\frac{d r_{e d}}{d R}\right)^{2}\right\}
\end{aligned}
$$

At end-diastole we have $\mu=1, k=1$ and $W_{\text {Ied, }}$, so we can write

$$
\sigma_{\theta e d}-\sigma_{\text {red }}=2 W_{\text {Ied }}\left[\left(\frac{r_{e d}}{R} \frac{d \theta_{e d}}{d \Theta}\right)^{2}-\left(\frac{d r_{e d}}{d R}\right)^{2}\right]
$$

We introduce the following notations

$$
\begin{gathered}
f\left(r_{e d}\right)=\sigma_{r e d}\left(r_{e d}\right)+P_{o e d}+\int_{r e d}^{b e d} D_{r e d} d r_{e d} \\
\int_{a}^{b} D_{r} d r=\bar{D}_{r} h
\end{gathered}
$$

The last equation is obtained by applying the mean value theorem. By substituting eqn (19) into eqn (2) and integrating we get the following result

$$
\begin{aligned}
\sigma_{r}(r)=\left(P-P_{o}-\bar{D}_{r} h-f\left(a_{e d}\right)\right) F(r) \\
-P_{o}-\int_{r}^{b} D_{r} d r+f\left(r_{e d}\right)
\end{aligned}
$$




$$
-F(r)=\frac{\int_{r}^{b} W_{I}\left(\mu^{2}-\frac{1}{\mu^{2} k^{2}}\right) \frac{d r}{r}}{\int_{a}^{b} W_{I}\left(\mu^{2}-\frac{1}{\mu^{2} k^{2}}\right) \frac{d r}{r}}
$$

By applying the mean value theorem one can take $W_{I}$ outside the integral and simplify this term by considering that it is nearly equal in numerator and denominator. One should notice that this simplification is at the expense of introducing a new factor in eqn (23) that is difficult to evaluate directly because of the presence of the term $\bar{D}_{r} h$. By making use of the relation $d \mu / d r=(\mu-$ $\left.k \mu^{3}\right) / r$ we can then integrate eqn (24) to get

$$
-F(r)=\frac{\frac{C_{k}}{k}\left(\frac{1}{r^{2}}-\frac{1}{b^{2}}\right)+\ln \left(1+\frac{C_{k}}{k r^{2}}\right)-\ln \left(1+\frac{C_{k}}{k b^{2}}\right)}{\frac{C_{k}}{k}\left(\frac{1}{a^{2}}-\frac{1}{b^{2}}\right)+\ln \left(1+\frac{C_{k}}{k a^{2}}\right)-\ln \left(1+\frac{C_{k}}{k b^{2}}\right)}
$$

We notice that eqn (23) can be split in the form $\sigma_{r}(r)=\sigma_{r p}(r)+\sigma_{r a}(r)$ as follows

$$
\begin{gathered}
\sigma_{r p}(r)=\left(P-P_{o}-f_{1}\left(a_{e d}\right)\right) F(r)-P_{o}+f_{1}\left(r_{e d}\right) \\
\sigma_{r a}(r)=\left(-\bar{D}_{r} h-f_{2}\left(a_{e d}\right)\right) F(r)-\int_{r}^{b} D_{r} d r+f_{2}\left(r_{e d}\right)
\end{gathered}
$$

where $f\left(r_{e d}\right)=f_{1}\left(r_{e d}\right)+f_{2}\left(r_{e d}\right)$. Eqns (26), (27) shows how the stress in the myocardium be split into two components respectively corresponding to the external constraints (pressure) and to the active muscular fibres.

\subsection{Calculation of the circumferential stress}

We write again eqn (2) in the form

$$
\frac{d\left(r \sigma_{r}\right)}{d r}=\sigma_{\theta}-r D_{r}
$$

By substituting eqn (23) we get the result 


$$
\begin{array}{r}
\sigma_{\theta}(r)=\left(P-P_{o}-\bar{D}_{r} h-f\left(a_{e d}\right)\right) \frac{d}{d r}(r F(r)) \\
-P_{o}-\int_{r}^{b} D_{r} d r-\frac{d}{d r} f\left(r_{e d}\right)
\end{array}
$$

We notice that $d r_{e d} / d r=k\left(r / r_{e d}\right)$ according to eqn (7). Notice that we can also write $\sigma_{\theta}(r)=\sigma_{\theta p}(r)+\sigma_{\theta a}(r)$ similar to eqns (26) and (27).

\section{Linear elasticity}

The passage from non-linear elasticity to linear elasticity depends on the way $F(r)$ is approximated, eqns (23) and (29) remain the same. If we write $\ln (1+z) \approx$ $z$ in eqn (25) we get the approximate value $F_{a p p}(r)$ for $F(r)$

$$
-F_{a p p}(r)=\frac{a^{2}}{b^{2}-a^{2}}\left(\frac{b^{2}}{r^{2}}-1\right)
$$

By substituting eqn (30) into eqns (23) and (29) we get

$$
\begin{gathered}
\sigma_{r}^{\prime}(r)=\left(P-P_{o}-\bar{D}_{r} h-f\left(a_{e d}\right)\right) \frac{a^{2}}{b^{2}-a^{2}}\left(1-\frac{b^{2}}{r^{2}}\right) \\
-P_{o}-\int_{r}^{b} D_{r} d r-f\left(r_{e d}\right) \\
\sigma_{\theta}^{\prime}(r)=\left(P-P_{o}-\bar{D}_{r} h-f\left(a_{e d}\right)\right) \frac{a^{2}}{b^{2}-a^{2}}\left(1+\frac{b^{2}}{r^{2}}\right) \\
-P_{o}-\int_{r}^{b} D_{r} d r-\frac{d}{d r} f\left(r_{e d}\right)
\end{gathered}
$$

If we neglect the end-diastolic term $f\left(r_{e d}\right)$, the two quantities $\sigma_{r}^{\prime}+\int_{r}^{b} D_{r} d r$ and $\sigma^{\prime}{ }_{\theta}+\int_{r}^{b} D_{r} d r$ correspond to the stress calculated by using linear elasticity in [3-6]. We also notice that by using eqn (30), eqn (23) can be written in the forms

$$
\begin{array}{r}
\bar{D}_{r} h-P+P_{o}-f\left(a_{e d}\right) \approx\left(\frac{\partial W}{\partial I}\right)_{m}\left(\frac{d r_{e d}}{d R}\right)^{2} \times \\
\frac{2 C_{k}}{k^{2}}\left(\frac{1}{a^{2}}-\frac{1}{b^{2}}\right)
\end{array}
$$




$$
\begin{array}{r}
\bar{D}_{r} h-P+P_{o}-f\left(a_{e d}\right) \approx\left(\frac{\partial W}{\partial I}\right)_{m}\left(\frac{d r_{e d}}{d R}\right)^{2} \frac{2}{k} \times \\
\frac{V_{\omega}}{V+V_{\omega}}\left(\frac{V_{e d}-V}{V}\right)
\end{array}
$$

In these equations $(\partial W / \partial)_{m}$ is a value calculated by the mean value theorem by taking $\partial W / \partial$ out of the integral sign, $V_{\omega}=\pi L\left(b^{2}-a^{2}\right)$ is the volume of the myocardium assumed constant and $V_{e d}$ is the end-diastolic volume. By taking $W$ $=(G / 2)(I-3)$ with $G=(Y / 2) /(1+v)$ we get a result similar to the result given in [11] $(G=$ shear modulus, $Y=$ Young's modulus, $v=$ Poisson's ratio).

\section{Comparison of the results}

Data for applications were taken from table 2 of [12] where values of $V_{e d}, V_{\omega}$ and $V$ at end-systole are given for fourteen normal patients. Going from large elastic deformation to linear elasticity involves basically approximating the logarithmic term in the expression of $F(r)$ by using the approximation $\ln (1+z) \approx z$. We have consequently compared in fig. 3 the exact value $x$.
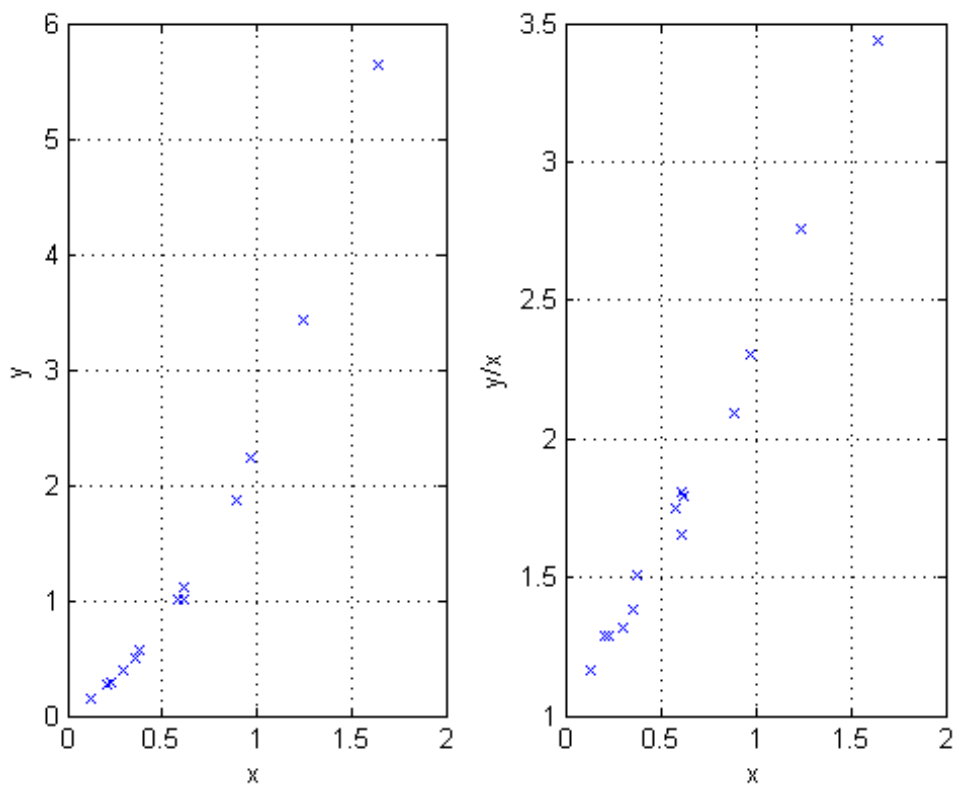

Figure 3: Left, plotting of the approximate value $y$ (eqn (36)) against the exact value $x$ (eqn (35)). Right, plotting of the ratio $y / x$ against $x$. Data taken from [12]. 
12 Modelling in Medicine and Biology VII

$$
x=\ln \left(\frac{b}{b_{e d}} \frac{a_{e d}}{a}\right)^{2}=\ln \frac{V_{e d}}{V_{e d}+V_{\omega}} \frac{V+V_{\omega}}{V}
$$

with its approximate value $y$

$$
y=\frac{C_{k}}{k}\left(\frac{1}{a^{2}}-\frac{1}{b^{2}}\right)=\frac{V_{\omega}}{V+V_{\omega}} \frac{V_{e d}-V}{V}
$$

It is seen that the ratio $y / x$ varies from nearly 1 to nearly 3.5 ; the greater $x$ the greater is the ratio. We have also compared in fig. 4 the exact value $x x$

$$
x x=\int_{a}^{b}\left(\mu^{2}-\frac{1}{\mu^{2} k^{2}}\right) \frac{d r}{r}
$$

or better to say its equivalent form
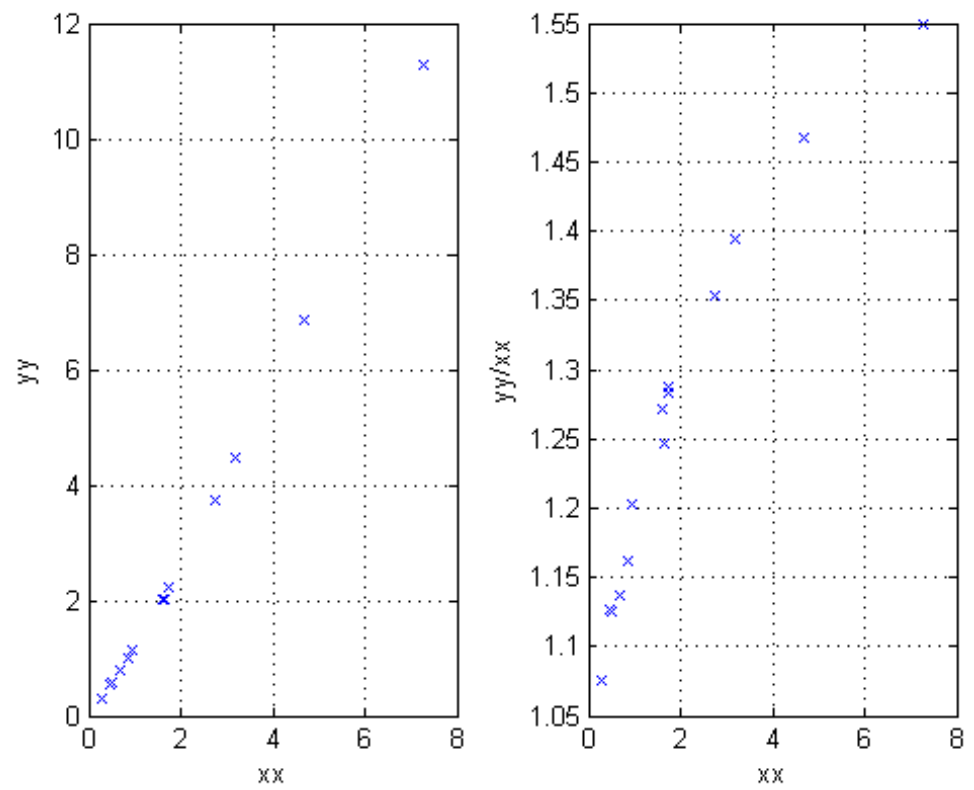

Figure 4: Left, plotting of the approximate value $y y$ (eqn (39)) against the exact value $x x$ (eqn (38)). Right, plotting of the ratio $y y / x x$ against $x x$. Data taken from [12]. 


$$
x x=\left(V_{e d}-V\right)\left(\frac{1}{V}-\frac{1}{V+V_{\omega}}\right)+\ln \frac{V_{e d}}{V_{e d}+V_{\omega}} \frac{V+V_{\omega}}{V}
$$

with its approximate value $y y$ taken from the right hand side of eqn (34)

$$
y y=2 \frac{V_{\omega}}{V+V_{\omega}} \frac{V_{e d}-V}{V}
$$

Eqns (38) and (39) enter in the expression of the radial stress calculated at endsystole on the inner surface of the myocardium. Fig. 4 shows that the ratio $y y / x x$ varies from 1.05 to 1.55 , indicating that the linear theory of elasticity can give results for the stress one time and a half greater than the results obtained by using large elastic deformation; the greater $x x$ the greater is the difference between the two values.

\section{Conclusions}

Calculation of the stress induced by the deformation of the passive medium of the myocardium by using the linear theory of elasticity has often given inconsistent results between theory and experiment, a review is given in [8].

Attempts to improve agreement between theory and experiment have led to assumptions that sometimes tend to complicate the problem. This study has presented a mathematical formalism that has allowed the derivation of the linear approximation of the stress from the expression of the stress obtained by using the theory of large elastic deformation. Results indicate what should be expected, the greater the deformation, the greater is the difference between non-linear and linear approximation.

\section{References}

[1] Shoucri, R.M., Theoretical study of pressure-volume relation in left ventricle, American Journal of Physiology, 260, pp. H282-H291, 1991.

[2] Shoucri, R.M., The pressure-volume relation and the mechanics of left ventricular contraction, Japanese Heart Journal, 31, pp. 713-729, 1990.

[3] Shoucri, R.M., Active and passive stresses in the myocardium, American Journal of Physiology, 279, pp. H2519-H2528, 2000.

[4] Shoucri, R.M., The calculation of the intramyocardial stress, Technology and Health Care, 10, pp. 11-22, 2002.

[5] Shoucri, R.M., The intramyocardial stress and the mechanics of the left ventricular contraction, Simulations in Biomedicine V, eds. Z.M. Arnez, C.A. Brebbia, F. Solina \& V. Stankovski, WIT Press: Southampton and Boston, pp. 177-187, 2003. 
[6] Mihailescu, S. \& Shoucri, R.M., Measurement and calculation of the intramyocardial stress, Modelling in Medicine and Biology VI, eds. M. Ursino, C.A. Brebbia, G. Pontrelli \& E. Magosso, WIT Press: Southampton and Boston, pp. 161-169, 2005.

[7] Shoucri, R.M., studying the mechanics of left ventricular contraction, IEEE Engineering in Medicine and Biology Magazine, 17, pp. 95-101, May/June 1998.

[8] Yin, F.C.P., Ventricular wall stress, Circulation Research, 49, pp. 829842, 1981.

[9] Spencer, A.J.M., Deformation of Fiber-Reinforced Materials, Clarendon Press: Oxford, UK. 1972.

[10] Simon, B.R., Kobayashi, A.S., Strandness, D.E. \& Wiederhielm, C.A., Large deformation analysis of the arterial cross section, ASME Journal of Basic Engineering, 93, pp. 138-146, 1971.

[11] Chu, B.M. \& Oka, S., Influence of longitudinal tethering on the tension in thick-walled blood vessels in equilibrium, Biorheology, 10, pp. 517-525, 1973.

[12] Gunther, S. \& Grossman, W., Determinants of ventricular function in pressure-overload hypertrophy in man, Circulation, 59, pp. 679-688, 1979. 